\title{
ASSOCIATION OF ACUTE APPENDICITIS WITH TYPICAL CLINIC AND ATYPICAL LOCATION DUE TO MALROTATION OF COLON, AND HYDROCEPHALUS /CASE REPORT/
}

\author{
Ludmil M. Veltchev ${ }^{1}$, Krassimir M. Iliev², Ognian N. Vassilev² \\ 1) Fellow, Master's Program in Hepatobiliary Pancreatic Surgery, Henri Bismuth \\ Hepatobiliary Institute; 12-14, avenue Paul Vaillant-Couturier, 94804 Villejuif \\ Cedex, France \\ 2) Deparment of general surgery, State hospital of Biala Slatina, Bulgaria
}

\section{SUMMARY}

Atypical appendix location due to malrotation of colon may suggest many different inflammatory diseases and may provoke physician's misdiagnosis. In the reported case the clinical findings suggested typical right lower quadrant symptomatology for appendicitis but the cecum and acutely inflamed appendix were located in the epigastria. Association of congenital anomalies such as hydrocephalus and malrotation of the cecum are found.

\section{Report of case}

Patient hospital \#2455

State hospital of B.SL, Bulgaria

A 12- year- old girl lived in facility for children with mental disorders and congenital anomalies, presented in July 09, 2009 in emergency room with generalized pain localized in upper abdominal cavity since 48 hours and with nausea and doubly vomiting.

Regularly presence of defecation, without of diarrhea.

She was admitted in surgical department for observation and diagnosis.

On admission, a girl in damaged status, with difficult mobility.

Irregular, minimal enlarged head with signs for hydrocephaly.

Neurologic status-mild mental retardation.

Abdomen: generalized abdominal tenderness was noted, most pronounced in the right lower quadrant, with definite rebound tenderness in this area.

An operative cicatrix, located in upper abdominal third was found from previous operative intervention-CSF shunting.

Blood analysis: Leukocytes numbered-18,300; Erytrocytes-4, 62; Hemoglobin contain was $-131 \mathrm{~g} / \mathrm{L}$. There were trace reactions for protein and acetone in urine analysis.

The next two hours, the rebound tenderness had become localized in the right lower quadrant and a diagnosis of appendicitis was made.
After preoperative intravenous antibiotic prophylactic dose, operation started with Roux incision and $500 \mathrm{ml}$ serous liquid was founded. Peritoneum presented hyperemia and fibrin. The small bowel was distended. In attempt to find and palpate right descending colon and appendix, neither one nor the other organ was in its normal anatomy location.

We decided to enlarge incisional opening vertically, via right pararectal abdominal narrowing. The distended small bowel was followed just until the cecum and we found appendix located under the inferior surface of the left hemiliver. No found another intraabdominal organ dislocation or malformation. The anomaly was that of failure of rotation of the bowel because in control observation in right hypochondria was found small fibrous bride fixing cecum and right lateral peritoneum. Peritoneal fluid was tacked and sent for microbiological culture-no organism grew in 72 hours. Appendectomy was carried out and the wound was closed in the usual manner. Antibiotic was extended to 4 days. In postoperative day eight without surgical complications, patient was discharged.

\section{DISCUSSION:}

Our case report presents interest because of association of two very rare congenital malformations such as malrotation of cecum and hydrocephalus. After literature search in Pub Med with words appendicitis, malrotation of cecum and hydrocephalus did not found any results.

Intestinal malrotation occurs at a rate of 1 in 500 live births. Most infants with gastroschisis, omphalocele, or congenital diaphragmatic hernia present with intestinal malrotation. Approximately $50 \%$ of patients with duodenal atresia and $33 \%$ of patients with jejunoileal atresia have a malrotation as well. Also, intestinal malrotation occurs in association with Hirschsprung disease, gastroesophageal reflux, intussusception, persistent cloaca, anorectal malformations (imperforate anus), and extrahepatic anomalies. 
The cause of intestinal malrotation is disruption in the normal embryological development of the bowel. Clinical features depend on the stage of disruption. Malrotation results from a failure of the gastrointestinal tract to complete its normal rotation as it returns to the abdominal cavity at 8-10 weeks' gestation. The bowel develops outside of the abdominal cavity as a single long loop of bowel based on the pedicle of the superior mesenteric vessels. As the bowel returns to the abdomen, the proximal small bowel returns first and the duodenum rotates underneath the superior mesenteric vessels to assume a retroperitoneal position. Rotation continues as the large bowel returns to the peritoneal cavity, rotating over the vascular pedicle to place the ileocecal valve in the right lower quadrant and establishing the hepatic and splenic flexures.

In malrotation, arrest of development can be characterized by an incompletely rotated cecum, with the result that it remains in a subhepatic position .Ectopic position of cecum is associated with atypical subhepatic location of appendix $(\mathbf{1}, \mathbf{2}, \mathbf{3}, \mathbf{4})$.

Hydrocephalus is usually due to blockage of CSF outflow in the ventricles or in the subarachnoid space over the brain. Occurs in 1/500 live births. Compression of the brain by the accumulating fluid eventually may cause convulsions and mental retardation (5). These signs occur sooner in adults, whose skulls no longer are able to expand to accommodate the increasing fluid volume within. Fetuses, infants, and young children with hydrocephalus typically have an abnormally large head, excluding the face. Hydrocephalus was treated with the surgical placement of a shunt system. Shunts divert the flow of cerebrospinal fluid (CSF) from a site within the central nervous system (CNS) to another area of the body, where it can be absorbed as part of the circulatory process.

\section{CONCLUSION}

No association was found in literature between intestinal malrotation and hydrocephalus.

It is rare combination of two different embryological malformations. When hydrocephalus appears immediately after birth and shunting is life threatening operation, none descending of cecum and appendix can be diagnosed only after prophylactical imaging study or in emergency of cause the appendiceal inflammation and laparotomy.

\section{REFERENCES:}

1. Chrysikopoulos H.Idiopathic normal pressure hydrocephalus: Thoughts on etiology and pathophysiology.Med Hypotheses. 2009 Jul 27. [Epub ahead of print]PMID: 19640656

2. Vechvitvarakul S, Harris BH. Appendicitis and malrotation.J Pediatr Surg. 2007 May; 42(5):905-6. PMID: 17502210

3. Zarzosa Hernández G, Pacheco Martínez P, Fernández Escudero B, García
Llorente C, Fernández Cebrián JM, Loinaz Segurola C, Quintans Rodríguez A. Intestinal malrotation in adults and acute appendicitis Rev Esp Enferm Dig. 2007 Sep; 99(9):559-60. Spanish. No abstract available. PMID: 18052657

4. Teyssedou C, Bigot P, Arnaud JP. Acute appendicitis in a patient with intestinal malrotation: the importance of CT imaging. J Chir (Paris). 2008 Nov-Dec; 145(6):601-2. PMID: 19106893
5. N. M. Shapiro,L.M.Michels, , S.Hurwitz. Appendicitis with typical symptoms but ectopic appendix due to malrotation of colon. Arch Surg 1963, 98; 3:158-159.

6. Chrysikopoulos H.Idiopathic normal pressure hydrocephalus: Thoughts on etiology and pathophysiology.Med Hypotheses. 2009 Jul 27. [Epub ahead of print]PMID: 19640656.
Corresponding author:

Ludmil Marinov Veltchev, MD PhD

Mobile: +359 876259685

E-mail: drlmarinov@yahoo.com 